\title{
Arthur Kaufmann
}

\section{Rechtsphilosophie}

C.H. Beck Verlag, München, 1997, 365 S., DM 64,--

Rechtsphilosophie - ein wenig beackertes Feld, aber nichtsdestoweniger wichtiges Betätigungsgebiet für die Jurisprudenz und die Philosophie. Immer wieder gibt es Hinweise auf das Bedürfnis beider Wissenschaften, zueinander zu finden - so manche Rechtsentscheidung ist nämlich eigentlich eine Moralentscheidung, denkt man etwa an höchstrichterliche Entscheidungen zum Abtreibungsrecht, zu Castor-Transporten oder etwa im Bereich der Landwirtschaft zur Massentierhaltung. Und so liegt es also nahe, die "Schnittmenge" zwischen Rechtswissenschaft und Philosophie aus der Perspektive juristischen Denkens in Hinblick auf die "Richtigkeit" der Aussagen im Bereich der Logik - die Grunddisziplin der Philosophie schlechthin - aufzusuchen, und etwa die Entscheidungen, die sich sozusagen auf das Handeln der Menschen beziehen, im Bereich der Ethik aufzusuchen. So weit so gut: Ein verheißungsvoller Versuch liegt vor: Kaufmanns "Rechtsphilosophie".

Kaufmanns Buch umfaßt zwanzig Kapitel. Nach einigen einleitenden Bemerkungen über die Aufgaben der Rechtsphilosophie im Allgemeinen (S. 7-9), zum Begriffe selbst (S. 11 16), folgen Ausführungen zur Rechtsgeschichte. Dabei findet die Rechtsgeschichte des Altertums (Platons "Politeia" und "Nomoi" (Gesetze) oder etwa das gesamte römische Recht) unter einer gesonderten Überschrift ebenso auf einer einzigen Seite (S. 22) Platz wie etwa die Rechtsgeschichte des Mittelalters (S. 23) oder die der Neuzeit (S. 24). Im Anschluß daran folgt ein "collegium logicum", das sich ausschließlich mit der zweiwertigen Logik beschäftigt. Andere Logiksysteme finden keine Erwähnung. Ein ausführliches "rechtliches" Kapitel befaßt sich mit juristischen Elementarbegriffen wie etwa "Satzung, öffentliches Recht, Privatrecht, Person, Zurechnung, Gerechtigkeit und Billigkeit" und könnte in seiner konvolutartigen Zusammenstellung vielleicht als Einleitung in das juristische Denken für Nichtjuristen gedacht sein (S. 94-161). Die Diskussion einer "Idee des Rechts" hebt an mit der Gegenüberstellung einer kommunistischen oder christlichen Sozialisation, wobei sich unmittelbar die Frage aufdrängt, ob das die beiden einzigen möglichen Sozialisationsformen auf dieser Welt sind. Die Abhandlung schließt mit Überlegungen zur Risikogesellschaft und Ökologie, Bioethik oder etwa "Toleranz - die Schicksalsfrage" und ähnlichen weltumspannenden Gedanken.

Viele Aspekte zu vielen Themen, die Kaufmann anspricht, muten recht interessant an, doch stellt sich beim Lesen so vieler verschiedener Gedanken die Frage nach dem didaktischen Leitfaden eines Lehrbuches zur Rechtsphilosophie. In einem solchen würde man vielleicht zunächst eine ausführliche Begriffserklärung erwarten, eine gründliche Erörterung der Geschichte der Rechtsphilosophie könnte folgen, ein interdisziplinäres Kapitel über die Auseinandersetzung zwischen Rechtswissenschaft und Philosophie könnte das Werk abrunden, bevor es in eine aktuelle Diskussion um verschiedene Standpunkte der Rechtsphilosophie einmündet. 
Doch über Philosophie spricht der Autor ebensowenig, wie er über das Recht selbst philosophiert. Allerdings hat Kaufmann - und dies ist symptomatisch für des Autors Umgang mit der Philosophie in dem gesamten Buch - sogleich an den Beginn seines Werkes ein "philosophisches Motto" gestellt: seine eigene Formulierung des kantischen "kategorischen Imperativs". Er nennt ihn den kategorischen Imperativ "der Toleranz" und formuliert ihn so: "Handle so, daß die Folgen deiner Handlung verträglich sind mit der größtmöglichen Vermeidung oder Verminderung menschlichen Elends". Kant selbst nennt den kategorischen Imperativ wie folgt: "Handle nur nach derjenigen Maxime, durch die du zugleich wollen kannst, daß sie ein allgemeines Gesetz werde." Vergleicht man beide Sätze einmal nach Inhalt und logischer Qualität, fällt sofort - geht man Schritt für Schritt die Formulierungen durch - einiges Bemerkenswerte auf:

Kants Formulierung ("Handle nur ...") setzt Allgemeingültigkeit voraus, Kaufmanns Formulierung hingegen ("Handle so,...") ist relativ.

Kant bezieht sich in seiner Formulierung: "nach derjenigen Maxime" auf das von der Vernunft als moralisch eindeutig Erkannte. Danach ist z. B. der Fall nicht eindeutig, in dem jemand nach einer Naturkatastrophe den Opfern Geld spendet, um sein Image aufzupolieren. Eindeutiger wäre der Fall, wenn jemand den Opfern Geld spendete, weil er der Auffassung wäre, man müsse möglichst jedem Menschen, der in Not ist, nach eigenen Möglichkeiten helfen. Kant betrachtet also nicht allein die Folgen einer Handlung (teleologischer Ansatz), sondern vor allem die Motivation einer Handlung, die sich aus der transzendentalen Sphäre des allgemeinen Sittengesetzes herleiten läßt (deontologisch). Kaufmann läßt bei der Formulierung seines "kategorischen Imperativs" dieses Grundprinzip, das man auf jeden Fall berücksichtigen sollte, da sonst die Verwendung des Kategoriebegriffes völlig sinnlos ist, unberücksichtigt.

Des weiteren stellt Kant klar, daß ein allgemeines Gesetz, also ein Gesetz, an das sich (Thomas v. Aquin würde sagen) jedes Geistwesen halten muß, zumindest evident, logisch, begründet sein muß ("durch die du zugleich wollen kannst, daß sie ein allgemeines Gesetz werde"). Dies ist die Folie, auf der Kant die Maximen jeglichen Handelns prüft und zwar nach Qualität, Quantität und Relation usw.. Kaufmann dagegen reduziert die Handlung rein auf ihre Quantität - nämlich "Vergrößerung oder Verminderung". Diese Reduktion auf einen Aspekt unter vielen ist im Sinne eines kategorischen Imperativs durchaus unlogisch. Wäre die Formulierung Kaufmanns ("menschlichen Elends") wenigstens als synthetischer "Imperativ" überhaupt logisch möglich, so könnten demnach etwa die Handlungen der Herrscher eines totalitären Regimes dann moralisch als "gut" bewertet werden, wenn das moralische Kalkül - darüber kann man übrigens sehr anschaulich etwas in den Schriften Jeremy Benthams nachlesen - ergibt, daß die Anzahl der Menschen, die "weniger Elend" haben, geringer wäre, wenn es die Terrorhandlungen dieses Regimes nicht gäbe. Wenn man dem "Imperativ" in Kaufmanns Rechtsphilosophie folgen wollte, ließe sich staatlicher Terror geradezu problemlos rechtfertigen.

Diese Neuformulierung des kategorischen Imperativs ist in dem vorliegenden Werk wohl der einzige ernstzunehmende Versuch mit der Philosophie selbst. Ansonsten kommt das 
Werk über allgemeine, individuelle Überlegungen zu diesem und jenem die Welt bewegenden Thema an kaum einer Stelle hinaus. Formulierungen wie: "Doch das erkenntnistheoretische Subjekt-Objekt-Schema gehört der Vergangenheit an, dies sogar in den erklärenden Naturwissenschaften, um wie viel mehr in den verstehenden Verhaltenswissenschaften." oder etwa "Und sind Atom-, Bio-, Gentechnologie etwa keine Probleme des bonum commune? (...) es ist erstaunlich, wie wenig sich Rechtsphilosophen dem Thema "Frieden" gewidmet haben." (S. 9) wirken eher plakativ.

Das Urteil des Autors, daß "Hermeneutik" der Gegner von "juristischer Argumentation" sei (S. 46), mutet befremdend an, da doch gerade die Hermeneutik als eine wissenschaftliche, erkenntnistheoretische Methode gut geeignet wäre, sich dem Recht als Phänomen menschlicher Kommunikation zuzuwenden. Wenn der Autor feststellt: "Nicht Pluralismus ist Hindernis bei der Wahrheitsfindung, sondern er ist eine höchst wichtige Voraussetzung, um zur Wahrheit zu kommen, freilich nicht zu einer 'objektiven' Wahrheit (die wir nicht erreichen können - nur die Kirche kann sie auf ihre Weise beanspruchen, weshalb auch nur sie mit innerem Grund anti-pluralistisch ist) aber doch zu einer intersubjektiven Wahrheit." (S. 48) bleiben die Antworten auf Fragen wie: Welche Kirche der Autor denn nun meint, oder: Was ist Wahrheit? offen. Wenn der Autor etwa über Recht als Kommunikation unter Menschen spricht, dann versteht er unter Kommunikation ausschließlich Strafrecht. Dabei sind . diejenigen, die überhaupt im juristischen Umfeld kommunizieren nach Kaufmanns Ansicht vor allem Richter und Täter (S. 191). Eine allein komplementäre Kommunikationssituation dürfte im Rahmen eines rechtsphilosophischen Diskurses eher mit erheblichen Mängeln behaftet sein. Verwendet der Autor zentrale philosophische Begriffe wie etwa "Wertphilosophie", werden nicht, wie zu erwarten wäre, in gleichem Atemzuge die Urheber der Begriffe - hier: Max Scheler oder Nikolai Hartmann - und die entsprechenden Fundstellen genannt, sondern Kaufmann zitiert sich, wie fast auf jeder Seite des Werkes, dazu selbst. Dabei würde die Originalzitation dem an Rechtsphilosophie Interessierten den Weg leichter eröffnen, sich der primären Literatur zuzuwenden. Kaufmann fokussiert schließlich die Schnittstelle zwischen Philosophie und Rechtswissenschaft auf die gesellschaftspolitische Frage nach sozialer Gerechtigkeit und bemerkt, ohne in seinem Buch etwas über die Rechtsphilosophie als solche gesagt zu haben oder eine Lehre der Rechtsphilosophie vorgestellt zu haben, abschließend dazu: "Was die besonderen Fragen zur sozialen Gerechtigkeit angeht, so läßt sich eine vollständige Darstellung nicht erreichen" (S. 185).

Resümee: "si tacuisset philosophus mansisset!

Guntram Platter 